\title{
Providing Connectivity to Implanted Electronics Devices: Experimental Results on Optical Communications over Biological Tissues with Comparisons against UWB
}

\author{
Senjuti Halder, Mariella Särestöniemi, Iqrar Ahmed, Marcos Katz \\ Centre for Wireless Communications \\ University of Oulu, Finland \\ senjutihalder@yahoo.com
}

\begin{abstract}
Radio and acoustic waves have been conventionally used for transmitting information through biological tissues. However, some radio-based communications often suffer from several drawbacks like security, safety, privacy, and interference. In this paper, we demonstrate that optical wireless communications can be practically used for communications through biological tissues, particularly to transmit information to and from implanted devices. In the experiment, ex vivo samples of pork meat were used as the optical channel. Initial results show that information can be optically transmitted through biological tissues to distances of several centimeters, a range of practical interest as many implants today are placed within this extent. Optical links are inherently secure, and interference to and from other equipment is not an issue. With numerous potential benefits, optical wireless communication can be considered as a complementary approach to the existing radio frequency (RF) communications. In this paper, a comparison between the measurement results of ultra-wideband (UWB) and optical communications through the biological tissues is presented. Both experiments have been taken place in a similar environment, with the same meat samples. We have also explored the effect of tissue temperature on successful communications through biological tissues. These initial results are very promising and indicate various potential benefits for in-body communication in the future.
\end{abstract}

Keywords: medical implants, optical communications, ultra-wideband, biological tissues, in-body communications.

\section{$1 \quad$ Introduction}

Improving the quality of life is one of the ultimate goals of technology, and in this respect, wireless and mobile communications play a key role. In particular, remote health monitoring is a cost-effective approach to the prevention of diseases as well as the provision of health care. The aging population, chronic diseases, and the desire to live a healthy lifestyle are present-day healthcare challenges that can be addressed by wireless technology. The concept of wireless body area networks (WBAN) is a wellknown example of this technology, where a network of nodes (e.g., sensors) inside, outside, and on the patient's body collects, processes, and transmits physiological data. Last decades have witnessed a remarkable development in electronic body implant 
technology. Today, we rely on a great variety of these implants, such as heart pacemakers, cardioverter defibrillators, medicine dispensers, and brain stimulation implants, for example. In addition, in-body sensors and smart pills have been developed to monitor physiological parameters, imaging, drug delivery, etc. Furthermore, there is currently an increasing interest in brain implants beyond conventional brain stimulation and deep brain stimulation. These include monitoring and recording brain activity and ultimately interfacing neural systems with computer systems (e.g., brain-computer interfaces). Recently, the idea of the internet of things (IoT) has been extended to consider in-body, on-body, and out-body nodes networked to provide medical care. New concepts such as the internet of medical things (IoMT) and the internet of the human body (IHB) have been lately proposed.

One of the key enablers in the above-mentioned systems is human centric communications (HCC), a field that has been and continues to be widely investigated. HCC defines wireless communications taking place inside the human body, communication between internal and external nodes as well as communication across nodes on the body. Fig. 1 depicts the possible wireless links in HCC, where three types of nodes are shown, a) in-body nodes (IBN), b) on-body (OBN) nodes, and c) out-body or external nodes (EN). In this paper, the focus is on in-body communications, where wireless links are established between IBNs or between IBNs and OBNs. Typical nodes include electronic implants as well as ingestible or implanted sensors, for instance. Radio has been the dominant technology for in-body wireless communications, exploiting narrowband, wideband, and ultra-wideband concepts. Acoustic communication is also an interesting approach to communicating through biological tissue. Human tissue is a challenging medium for the propagation of radio waves. Radio frequency attenuation is typically high, due to the high conductivity and dielectric constant of the biological tissues. These characteristics strongly depend on the frequency used for communications. Moreover, the medium could be very complex, as the signal may need to propagate through many layers of different tissues, each with different characteristics. In-body communication must be highly secure and private, and this is a challenge for radio systems. Several cases have emerged in recent years, showing the vulnerability of commercial implants to hacks, leading even to a massive recall of implantable devices. Besides, the radio is prone to be affected by non-intentional interference. Ultrasound-based communication systems do not face these challenges, though they might be limited by low data support and considerably high-power consumption. In this paper, we propose the use of optical communications for establishing in-body wireless links. Previous work has shown that optical communication is viable for very short optical links of a few millimeters, particularly for reaching electronic devices placed under the skin. This is the case of superficial communications, indicated with "a" in Fig. 1. However, our initial experiments have shown that considerably longer ranges can be easily achieved using optical links based on near-infrared (NIR) light. This case, depicted with "b" in Fig. 1, provides connectivity to in-body electronic nodes (IBNs). Moreover, connecting in-body and out-body nodes (e.g., EN) is also possible using optical links. The prospect of using light for wireless communications inside the human body is highly relevant. Optical wireless communications have unique advantages such as high security, privacy, safety as well as low complexity and power consumption. Furthermore, light sources are used 
for a variety of novel purposes inside the human body, such as for diagnosis and therapy. Control of cellular activity (e.g., in neurons) by means of light to activate, inhibit, or monitor certain functions is another highly relevant application of light. The vision of having key health functionalities such as wireless communication, diagnosis, treatment, and monitoring working with under the same light-based infrastructure is truly appealing. In this paper, we report the design, implementation, and testing of an optical communication system, with a testbed using realistic ex vivo samples as the optical channel. The optical system is compared to an ultra-wideband communication system working in a similar scenario. The impact of the temperature on the optical and radio communication links is also investigated. There are several studies presenting the impact of the temperature on the dielectric properties of the animal tissues in different frequency ranges [1][2]. However, up to the authors' knowledge, there is a lack of studies presenting the impact of the temperature on the radio channel characteristics. In ref. [3], authors have presented results on the impact of the temperature on the antenna and channel characteristics. Frequency domain channel results are repeated in this paper to ease the comparison with the measurement results obtained with the optical technique at the same temperature.

This paper is organized as follows. Section 2 introduces optical communications through biological tissues, while Section 3 provides a comparison among radio, acoustic, and optical wireless systems for in-body communications. Section 4 presents measurement results from a realistic setup justifying the optical communication approach. Discussions on opportunities, challenges, and novel applications are presented in Section 5. Finally, Section 6 concludes the paper.

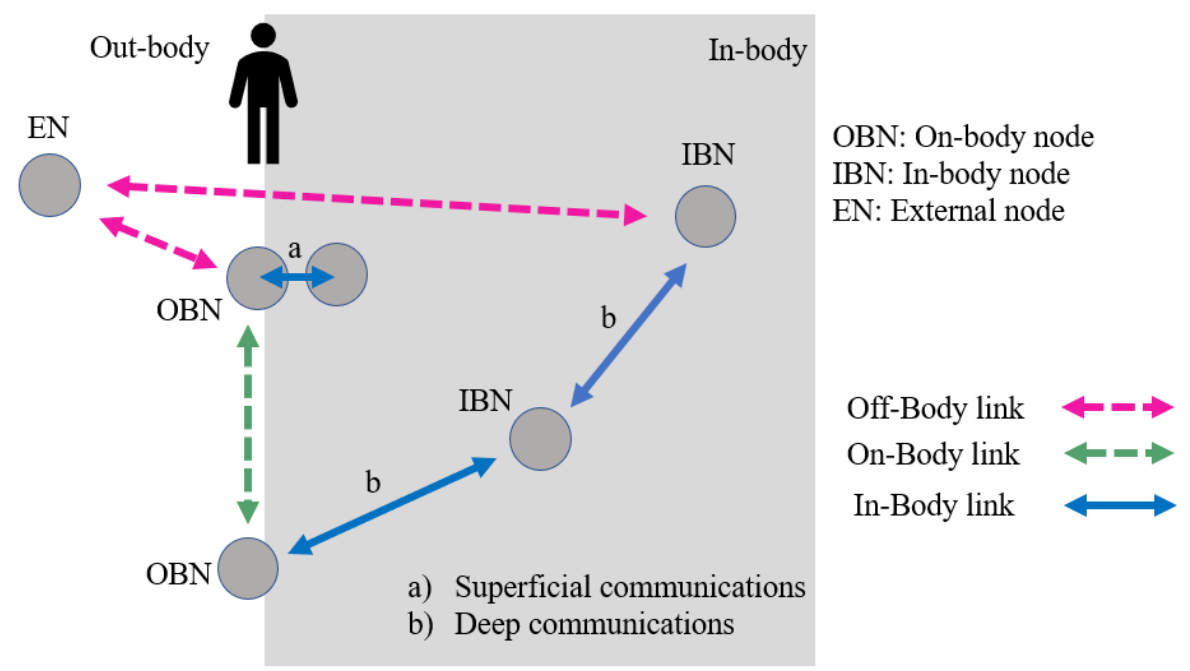

Fig. 1. HCC: In-body, out-body, and off-body links. 


\section{Optical Communications Through Biological Tissues}

Visible and infrared light is widely used in medical information and communication technology (ICT), mostly for diagnosis and therapeutics. In this section, we briefly introduce the basic ideas behind optical communications in biological tissues, in other words, how to exploit light to transmit information through biological tissues. Unlike conventional uses of optical wireless communications (OWC), where the medium is air or water, biological tissues are a challenging medium. Interaction of light with biological tissues is a well-studied field, particularly aiming at analyzing properties of the tissue and eventually making the diagnosis. However, biological tissues have not been studied from the wireless communications standpoint. Light is reflected, absorbed, scattered, and transmitted through biological tissues. Biological tissues are characterized by high anisotropy and strong absorption and scattering. The near-infrared window in the region (700-1100) $\mathrm{nm}$ is commonly used as light is less attenuated than in other wavelengths. Due to scattering, light is dispersed, reducing rapidly its energy as light propagates across the tissue. Light is thus highly attenuated, and pulses are spread in time. The receiver needs to cope with the signal impairments imposed by this severe medium in order to recover the transmitted signal. Contrasting air and water, one can expect that the range of optical links in tissues is quite limited, a fact that is in line with the geometry of the human body and the ranges demanded by typical in-body medical applications. Increasing the optical power of the transmitted signal can be used to increase the range or data rate. On the other hand, to avoid damages to the biological tissues, the energy and power density of the transmitted signal need not to exceed certain limits, defined by the International Commission on Non-Ionizing Radiation Protection [4].

Rayleigh and Mie scattering are commonly used to model the optical scattering process in biological tissues, where the former takes place when the scattering is caused by particles much smaller than the wavelength of light, and the latter when the sizes of particles are in the order or larger of the light wavelength.

\section{Radio, Acoustic and Optical Wireless Communications in Biological Tissues: A Comparison}

\subsection{Radio}

Radio technology has been widely used in medical applications for several decades. It has several advantages for implant communications. It can provide high-data-rate communications and, also high-resolution images using low transmit power. Especially with lower frequencies, power losses in the tissues are moderate. Radio is a well-known technique for which inexpensive components are widely available. Besides, the in-body data can easily be transmitted out of the body since most of the medical devices use RF [5][6].

Narrowband (NB) and UWB technologies are the most commonly used radio technologies in the medical applications based on the WBAN, which is considered as a 
collection of miniaturized low-power, wireless sensors for monitoring the human body functions [6]. For the successful implementation of WBAN applications, the IEEE published the standard 802.15.6 [7] for in-body/on-body node communications. In addition to the main technologies NB and UWB, WBAN covers also human body communications (HBC) technology, which is based on galvanic coupling or capacitive coupling. From these techniques, UWB is chosen to be compared with the optical communication technique.

UWB technology has been recognized as a promising candidate for implant communication systems. It provides several advantages: high data rates, low power consumption, high security, simple transceivers, etc. [6]. The main drawback of the UWB is the higher propagation loss within the tissues. However, the propagation loss can be dealt with the use of a lower UWB band (3-5 GHz) in the applications where the signal is required to propagate deep inside the body tissues and with properly directive antennas. UWB has been used in several medical monitoring applications, such as capsule endoscopy, tumor detection, heart and breath rate monitoring, glucose level monitoring, etc.

\subsection{Acoustic}

Mechanical movements of particles in a substance form the ultrasounds (US) wave [8]. Ultrasonic waves are generally defined by the following parameters: frequency, pressure, amplitude, propagation speed, and intensity [9]. US is an extensively popular technology in healthcare due to its various applications in imaging. Ultrasound consists of all the acoustic waves having a frequency above $20 \mathrm{KHz}$, which is non-audible for humans [10]. Apart from the numerous imaging applications, US has been considered to have great potential for in-body communications. Acoustic waves, typically generated through piezoelectric materials are preferred to be used for underwater communications, as they propagate better in media mostly made up of water when compared to RF. Up to $60 \%$ of the human adult body consists of water [11] and blood is a fluid that provides cells with different resources. This makes ultrasound an eligible technology to support in-body communications.

\subsection{Optical}

Over the years, there has been a growing interest in utilizing optical communications for the betterment of healthcare technologies. Light has been widely used in both therapeutic and diagnostic medical applications. Light-based communications typically require line-of-sight (LOS) configuration [12]. However, non-line-of-sight (NLOS) configuration is also possible, though the signal strength could be greatly affected in that scenario. In recent years, visible light communications (VLC), a case of OWC, has become an extremely popular research topic. In the electromagnetic spectrum, the visible spectrum expands over a wavelength of $400 \mathrm{~nm}$ to $700 \mathrm{~nm}$ [13]. In VLC, lightemitting diodes (LEDs) are employed due to their advantages of simultaneously providing illumination and transmitting of data. Optical communications possess numerous advantages like safety, large and unregulated spectrum, data security, less interference, zero-radio-exposure as well as cost and energy effectiveness. These advantages could lead these light-based communications a suitable choice for future medical 
applications. The utilization of optical communications in implants is very safe and secure for patients, a fundamental advantage of this technology. For OWC, IEEE published the standard 802.15.7 in 2011 which mainly aimed at visible light spectrum. Development work has been going on to achieve further standards so that the ultraviolet, visible light and infrared areas can also be included.

Some key characteristics of UWB, acoustic, and optical communications system are presented in Table 1.

Table 1. General comparison of UWB, acoustic and optical communications for short-range medical applications.

\begin{tabular}{llll}
\hline & Radio (UWB) & Acoustic & Optical \\
\hline Supported data rate & $0.5-10 \mathrm{Mbps}$ & $<0.5 \mathrm{bps}$ & $100 \mathrm{Kbps}$ \\
Range & $<10 \mathrm{~cm}$ (directive antennas) & $<15 \mathrm{~cm}$ & $<5 \mathrm{~cm}$ \\
Power requirements & Low & Low $/$ moderate & Potentially low \\
Complexity & Low & Low & Low \\
Security/ privacy & Low & High & Very high \\
Safety/ exposure & Safe within SAR limits & Safe & Safe \\
Standard & $802.15 .6,802.15 .4$ & None & None \\
Frequency & $3.1-10.6 \mathrm{GHz}$ & $1-3 \mathrm{MHz}$ & $300 \mathrm{GHz}-430 \mathrm{THz}$ \\
\hline
\end{tabular}

\section{$4 \quad$ Experimental Results}

In this section, we present some measurement results carried out with individual experimental testbeds for both optical and UWB. Results, though initial, show the potential of optical wireless communications for in-body applications.

\subsection{Optical Measurements}

The optical communication measurements have been carried out using mostly commercially available equipment. An experimental testbed was developed to evaluate optical communication through biological tissues [14]. The testbed consists of an optical transmitter, an optical receiver, and the biological tissue as the optical channel. Fig. 2 shows the basic block diagram of the testbed used in the measurement setup. The transmitter side comprises a computer, universal software radio peripheral (USRP) where the key blocks of the transmitter were implemented, bias-tee, LED driver, and LED source. Thorlabs DC2200 LED driver [15] and an $810 \mathrm{~nm}$ mounted IR LED produced by Thorlabs [16] are implemented in the transmitter side. On the other end, the optical receiver comprises a photodetector, USRP where receiver blocks were implemented and a computer. The photodetector used in the receiver side is a silicon avalanche photodetector APD120A from Thorlabs [17]. For both transmitter and receiver side, NI USRP-2920 have been used which are designed and developed Ettus Research, the daughter company of National Instruments. We have used NIR light to illuminate the 
biological tissue since NIR light propagates better through tissues. The tunnel between source and receiving nodes has been created with the help of GNU radio software. For the optical communication link through biological tissues, a Gaussian minimum-shift keying (GMSK) modulation was used. The GMSK modulation scheme has been used due to its constant envelope property. It is difficult for the LED to be able to respond to the prompt phase changes of some other modulation schemes (e.g., quadrature phaseshift keying (QPSK)). Thus, GMSK modulation is considered as a convenient choice for the optical communications as the phase changes occur steadily in GMSK [14],[18].

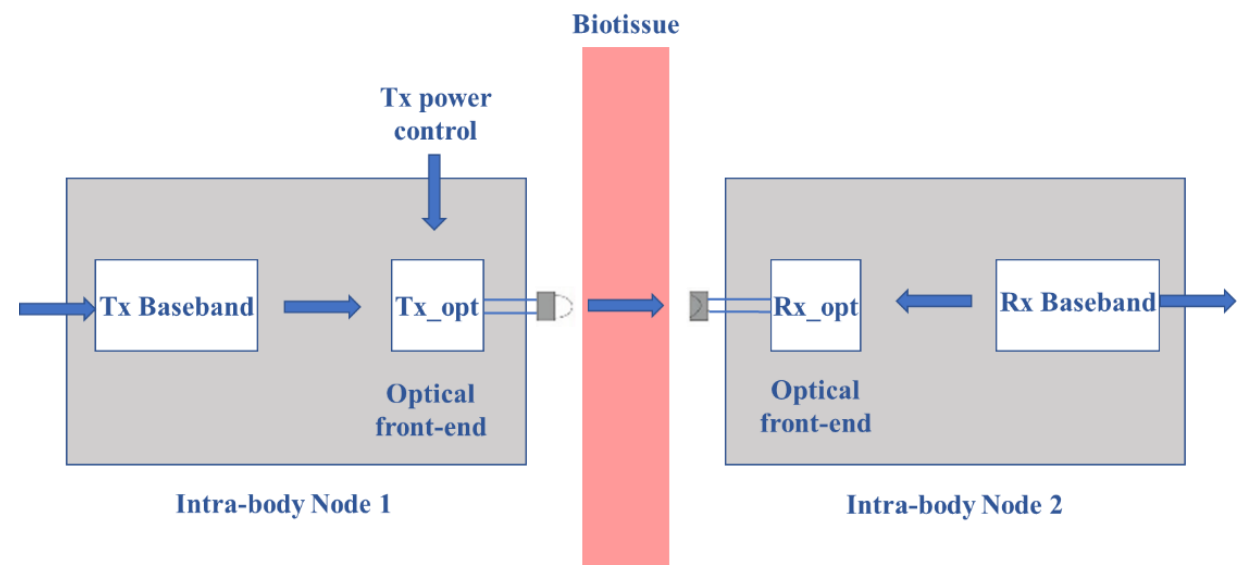

Fig. 2. Simplified block diagram of the experimental testbed.

Here, we have used a $3 \mathrm{~cm}$ thick fresh pork meat sample with the skin on. The meat sample consists of both muscle and fat (approximately $0.5 \mathrm{~cm}$ of fat and $2.5 \mathrm{~cm}$ of flesh). The temperature of the sample was $12^{\circ} \mathrm{C}$. The LED input current has been varied and we have measured the corresponding optical power employed on the meat sample. The optical powers were well in accordance with the standard of laser safety ANSI.Z136.1- 2007 [19]. Successful optical communication was established through the ex vivo sample. Moreover, an image file of $14 \mathrm{MB}$ has been transmitted through the sample and we were able to achieve a data rate of $22 \mathrm{Kbps}$. The meat sample has been illuminated on the skin surface and the detector has been placed on the flesh side. The experimental setup for optical communications through biological tissues is presented in Fig. 3. 

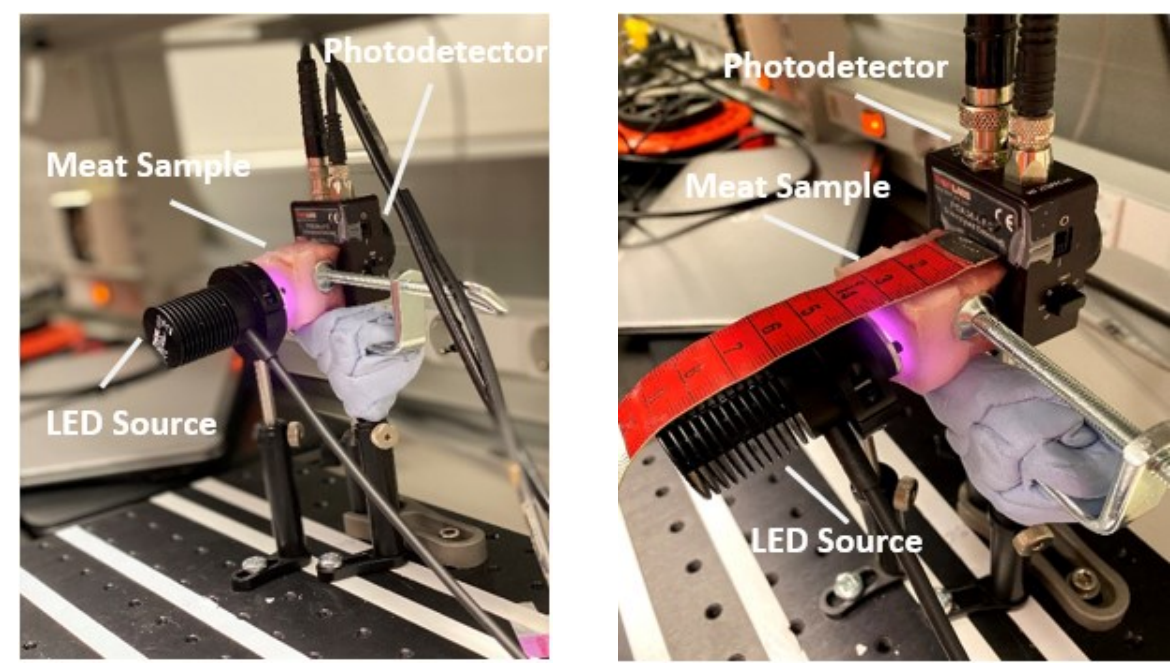

Fig. 3. Experimental setup for optical communications through biological tissue.

To understand the effect of tissue temperature on optical communication, the same meat sample has been heated to $37^{\circ} \mathrm{C}$. For this heating purpose, we have placed the sample inside a heating box made of acrylic material. A temperature controller was used to set the desired temperature of the sample. Table 2 presents the transmitted and received optical power for both cold and warm meat along with the power losses. Both the transmitted and received optical powers are measured with the help of PM100D power meter from Thorlabs [20].

Table 2. Transmitted optical power, received optical power and power losses of cold and warm meat.

\begin{tabular}{lclll}
\hline $\begin{array}{l}\text { Transmitted op- } \\
\text { tical power } \\
\left(\mathrm{mW} / \mathrm{cm}^{2}\right)\end{array}$ & $\begin{array}{l}\text { Received optical } \\
\text { power }(\mu \mathrm{W}) \\
\text { (Cold meat) }\end{array}$ & $\begin{array}{l}\text { Power loss }(\mathrm{dB}) \\
(\text { Cold meat) }\end{array}$ & $\begin{array}{l}\text { Received optical } \\
\text { power }(\mu \mathrm{W}) \\
\text { (Warm meat) }\end{array}$ & $\begin{array}{l}\text { Power loss }(\mathrm{dB}) \\
\text { (Warm meat) }\end{array}$ \\
\hline 50.94 & 98.3 & -27.14 & 128.3 & -25.98 \\
101.11 & 204.2 & -26.94 & 234.2 & -26.35 \\
146.26 & 301.8 & -26.85 & 341.8 & -26.31 \\
190.53 & 389.9 & -26.89 & 419.9 & -26.57 \\
231.38 & 469.2 & -26.92 & 520.2 & -26.48 \\
\hline
\end{tabular}

A comparison of the received optical power in both cases (cold and warm meat samples) shows a minor difference between the measured values. The received optical power for both cold and warm meat samples are presented in Fig. 4. The optical properties of the meat sample change with the temperature and this affects the amount of received power. Moreover, as our sample consisted of both flesh and fat, the fat portion has become more transparent due to the rise in the temperature. This transparency also leads to better light propagation through the tissues, as seen in Fig. 4. 


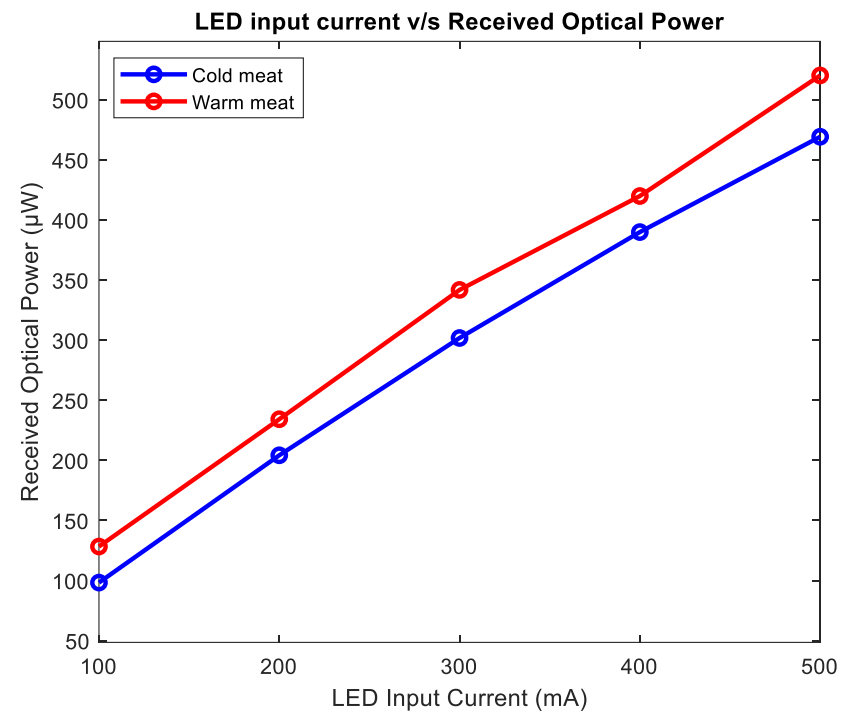

Fig. 4. LED input current $\mathrm{v} / \mathrm{s}$ received optical power.

\subsection{UWB measurements}

For a fair comparison, the UWB testbed used the very same meat sample used for the case of optical communication. UWB measurements were conducted using the Agilent 8720ES Vector Network Analyzer (VNA). Two different UWB-antennas were connected to the VNA's ports with coaxial cables. Antennas were placed on the top and below the biological tissue. The block diagram of the UWB measurement set up is presented in Fig. 5.

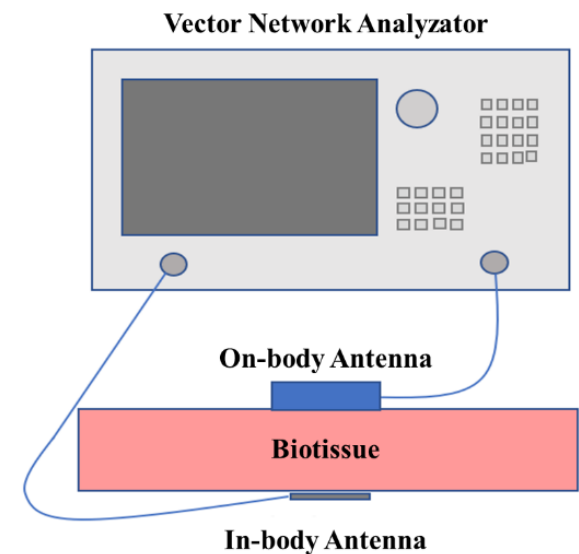

Fig. 5. Simplified block diagram of the UWB measurement setup. 
The UWB measurement results are analyzed in more detail in [3] presenting a study on the UWB in-body measurements using pork meat. Ref. [3] evaluates also the impact of the meat's fat and muscle composition as well as the impact of meat's temperature on the UWB antenna and channel characteristics and compares the UWB channel characteristics on humans and pork tissues by simulations. One of the channel results of [3] is represented in this paper to ease the comparison with the results obtained using optical communication.

Fig. 6 presents the meat used in the measurements. The size of the meat is $22 \mathrm{~cm} \mathrm{x}$ $20 \mathrm{~cm}$, which is much larger than that used in the optical measurements due to antenna sizes and the necessity to avoid propagation flow from the meat sides. The thickness of the meat is $3 \mathrm{~cm}$ as in optical measurements. The frequency-domain channel characteristics are evaluated for the UWB range of 0.5-5 GHz. Two different UWB antenna prototypes are used in the measurements: 1) a cavity-backed antenna, which is used as an on-body antenna and is located above the skin layer and 2) a loop antenna, which resembles as an in-body antenna. The cavity-backed antenna is a directive on-body antenna designed for implant communications at the frequency band $3.75-4.25 \mathrm{GHz}$, which is the frequency range of interest in this UWB measurement study. A detailed description of the antenna can be found in [21]. The loop antenna is an omnidirectional antenna designed to work for the whole UWB band 3.1-10.6 GHz. Details of the loop antenna can be found in [22].

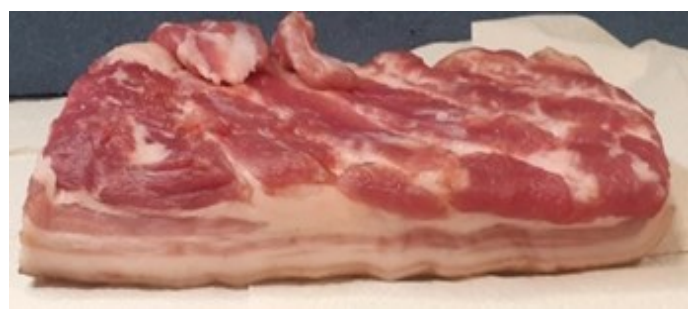

Fig. 6. The meat piece used in the measurements.

The meat piece was set inside a cube form area made by absorber pieces. The loop antenna was set below the meat muscle and fat tissue side. The cavity-backed antenna was set to the top of the tissues on the skin side. Fig. 7 presents the measurement setup, which is explained in detail in [3]. Ref. [3] also analyses in more detail the frequency and time domain channel characteristics as well as antenna characteristics with different pork meat pieces. This section summarizes the channel results for the pork meat sample which was also used in optical measurements to ease the comparison with optical measurements. The measurements were taken as the meat was cold $\left(12^{\circ} \mathrm{C}\right)$ and warm $\left(37^{\circ} \mathrm{C}\right)$, as in the optical measurements. 

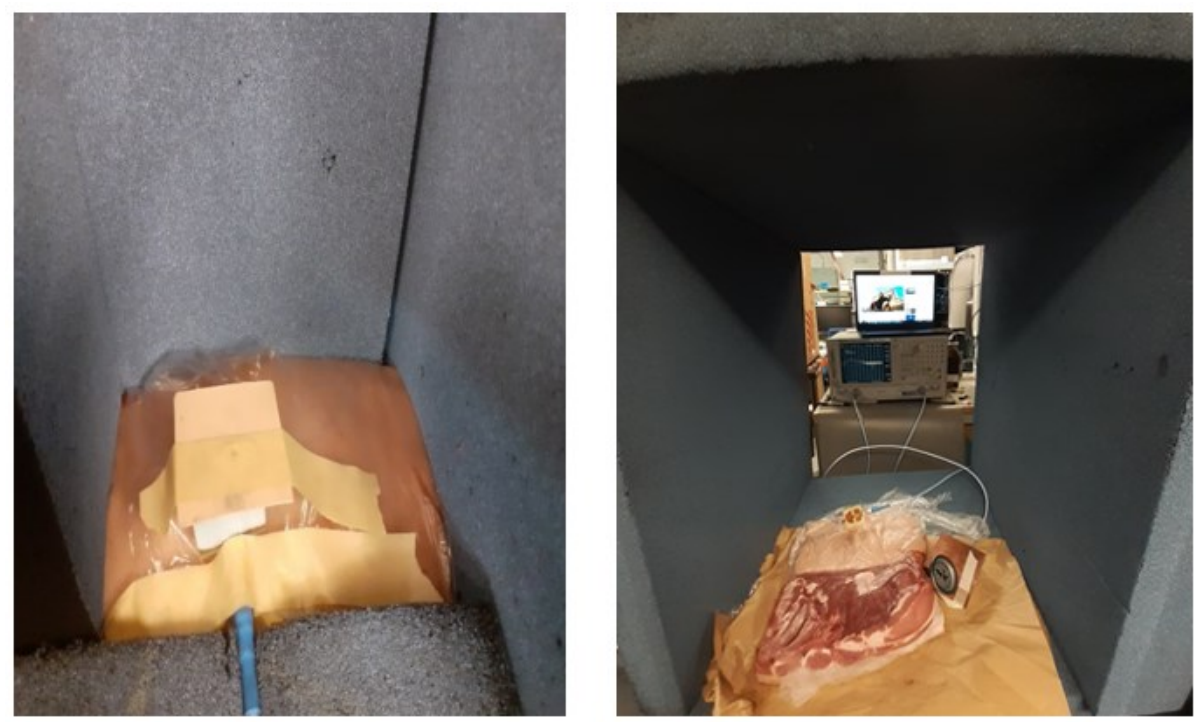

Fig. 7. UWB measurement setup inside the cube made from absorber pieces [3].

Frequency domain channel characteristics, i.e., S21 parameters, are presented in Fig. 8 for the pork meat with cold and warm temperatures. It can be seen, power loss on the frequency range of interest, i.e., $3.75-4.25 \mathrm{GHz}$, varies from $-48 \mathrm{~dB}$ to $-58 \mathrm{~dB}$ with the cold meat. The power loss difference at the $3.75 \mathrm{GHz}$ and $4.25 \mathrm{GHz}$ is noteworthy. The higher the frequency, the larger is the power loss due to changes in the dielectric properties [23]. Nevertheless, the power loss is at a reasonable level for successful data communications in this case. For the warm meat, the power loss is approximately $6 \mathrm{~dB}$ smaller compared to the cold meat. The variation within the frequency range of interest is -42 to $-52 \mathrm{~dB}$. These results show clearly, how the temperature has a clear impact on the channel characteristics since the dielectric properties of the tissues change with the temperature.

It can be concluded that the power loss for $3 \mathrm{~cm}$ thick meat remains at a reasonable level for a data communication link. With lower UWB frequencies and directive antennas, data communications might be possible even with double meat thickness in the realistic scenario of $37^{\circ} \mathrm{C}$ meat temperature.

The measurements have been taken with a meat piece having interlaced muscle and fat layers. Fat is known to be among the easiest tissues for radio propagations [24],[25] and thus the "fat tunnels" within the muscle layers help to achieve reasonable power loss level. With meat pieces having separate muscle and fat layers, the power loss is worse [3].

With the UWB technique, better propagation depth is obtained. The best propagation depth is achieved with directive on-body antennas. However, such antennas usually require relatively large cavity structures which might be clumsy for wearable devices. 


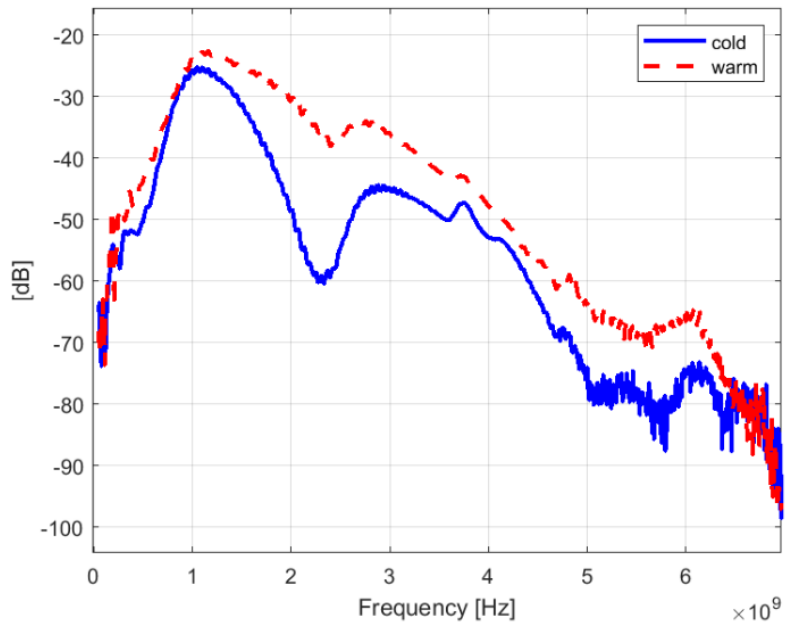

Fig. 8. The S21 parameter obtained in UWB measurements with the meat at the temperature of $12^{\circ} \mathrm{C}$ and $37^{\circ} \mathrm{C}[3]$

\section{Discussion}

This study investigates the potential of transmitting information through biological tissues by utilizing optical communications. Results show that optical communications through biological tissues are possible, in a range of practical interest. A comparison of the optical communications through biological tissues with the UWB communication is also presented in this paper. Here, we have used the same piece of meat sample with $3 \mathrm{~cm}$ thickness as the medium for both UWB and optical measurements. Individual experimental testbeds have been used to carry out the measurements. Light propagation through biological tissue is an extremely complex process, where phenomena like absorption, scattering, and reflectance take place during the propagation. The meat sample we have used here contains both fat and muscle. The optical power applied to the sample during the optical communications is well below the regulated safety limits. First, we have demonstrated that optical communications through biological tissues are feasible through ranges that are of practical interest, e.g., wireless connectivity to different implanted devices can be provided. In fact, in the future, very secure and safe communications could be provided to several possible implanted electronic devices such as pacemakers, defibrillators, insulin pumps, and others. The simple optical communications system was not matched to the optical channel, and thus, the supported data rate was relatively low, of the order of several tens of Kbps. Nevertheless, in most of the cases involving implanted devices, the required data throughput is relatively small, as typical applications require the sporadic transmission of sensor information, changing device settings, etc. High-order modulation schemes as well as multiple optical beams can be used to increase the data rate. 
An initial comparison between optical and UWB communications through biological tissues has been presented here. The comparison here focuses only on the attenuation of the two systems for a similar piece of sample. It was found that power loss with the optical system is minor than with UWB. However, also with UWB the loss is moderate and still far from the UWB receiver's sensitivity limits. Besides, the comparison is not straightforward since in the UWB measurement setup, only a pure sinusoid signal is inserted directly to the transmitter antenna without any modulation.

Optical and UWB measurements on the meat sample were carried out at $12^{\circ} \mathrm{C}$ and $37^{\circ} \mathrm{C}$ to observe the effect of tissue temperature during the communications. We have found that the received optical power of the heated meat sample is higher than that of the cold meat sample. Moreover, the power loss for the heated meat has been found to be smaller than the cold meat during UWB measurements. On the other hand, we have noticed minor differences in the power losses for the cold and warm meat during the optical measurements. So, it can be considered that optical communication is less sensitive to the tissue temperature compared to UWB communications. The measured power losses for both cold and warm samples are such that the signal can be decoded at the receiver.

Various individual advantages of optical and radio communications can be exploited together to implement a flexible hybrid optical-radio wireless network. In this hybrid optical-radio wireless network, both the optical and radio communications complement each other. An optical-radio hybrid network has several advantages, including increased link connection reliability, security, and support of higher data rates. Instead, the radio enables implant communication in the cases where the implant is deeper inside the tissues. The hybrid optical-radio network can be considered as a suitable approach for future medical applications due to these potential benefits.

\section{Conclusion}

In this paper, the feasibility of optical communications through biological tissues is investigated. A realistic scenario exploiting ex vivo samples is used in the study. A conventional UWB communication system is used as a baseline for comparison. The initial results regarding optical communications through biological tissues are very encouraging. Although the data rate achieved for optical communications is relatively low, it could well be sufficient for most of the electronic medical implants in use today. A range of three centimeters was obtained for the optical link, a distance well suited to provide connectivity to prevailing implants such as pacemakers, defibrillators and implanted drug dosing systems, for instance. Conventional narrow-band radio-based systems often face challenges like security, privacy, and safety, which need to be seriously tackled in any medical-related application. Both optical communication system as well as UWB based radio technique inherently overcome these drawbacks. The benefit of optical communication system over UWB is smaller device size and minor power loss. However, despite of minor power loss, optical communication is restricted only to few centimeters whereas with directive antennas at lower parts of the UWB range, the reliable communication can be achieved even up to $10 \mathrm{~cm}$. 
Optical communication can either be utilized as a standalone approach or in combination with other conventional communications (e.g., acoustic/radio). These advantages of optical communications indicate that it can be considered as a suitable approach for different future medical applications. In the future, extensive measurements will be carried out to achieve a more comprehensive comparison between the optical and UWB systems. The connectivity to the implanted electronic devices using optical and UWB communications will be further investigated where both the communication system would transmit data through biological tissues.

\section{$7 \quad$ References}

[1] Ley, S., Schilling, S., Fiser, O., Vrba, J., Sachs, J., \& Helbig, M. (2019). Ultra-wideband temperature dependent dielectric spectroscopy of porcine tissue and blood in the microwave frequency range. Sensors, 19(7), 1707.

[2] Gabriel, C., Gabriel, S., \& Corthout, Y. E. (1996). The dielectric properties of biological tissues: I. Literature survey. Physics in medicine \& biology, 41(11), 2231.

[3] M. Särestöniemi, Carlos Pomalaza-Raez, Chaimaa Kissi, J. Iinatti, "On the UWB in-body propagation measurements with a pork meat," submitted to BodyNets2020.

[4] International Commission on Non-Ionizing Radiation Protection. (2000). ICNIRP statement on light-emitting diodes (LEDs) and laser diodes: implications for hazard assessment. Health Physics, 78(6), 744-752.

[5] Teshome, A. K., Kibret, B., \& Lai, D. T. (2018). A review of implant communication technology in WBAN: Progress and challenges. IEEE reviews in biomedical engineering, 12, 88-99.

[6] Movassaghi, S., Abolhasan, M., Lipman, J., Smith, D., \& Jamalipour, A. (2014). Wireless body area networks: A survey. IEEE Communications surveys \& tutorials, 16(3), 16581686.

[7] IEEE Standard for Local and metropolitan area networks - Part 15.6: Wireless Body Area Networks." IEEE.

[8] Davilis, Y., Kalis, A., \& Ifantis, A. (2010). On the use of ultrasonic waves as a communications medium in biosensor networks. IEEE Transactions on Information Technology in Biomedicine, 14(3), 650-656.

[9] Galluccio, L., Melodia, T., Palazzo, S., \& Santagati, G. E. (2012, January). Challenges and implications of using ultrasonic communications in intra-body area networks. In 2012 9th Annual Conference on Wireless On-Demand Network Systems and Services (WONS) (pp. 182-189). IEEE.

[10] Carovac, A., Smajlovic, F., \& Junuzovic, D. (2011). Application of ultrasound in medicine. Acta Informatica Medica, 19(3), 168.

[11] Jéquier, E., \& Constant, F. (2010). Water as an essential nutrient: the physiological basis of hydration. European journal of clinical nutrition, 64(2), 115-123.

[12] Cevik, T., \& Yilmaz, S. (2015). An overview of visible light communication systems. arXiv preprint arXiv:1512.03568.

[13] J. C. Zwinkels and C. Canada, "Encyclopedia of Color Science and Technology," Encycl. Color Sci. Technol., no. January 2015, 2019. 
[14] Ahmed, I., Bykov, A., Popov, A., Meglinski, I., \& Katz, M. (2019, October). Optical Wireless Data Transfer Through Biotissues: Practical Evidence and Initial Results. In EAI International Conference on Body Area Networks (pp. 191-205). Springer, Cham.

[15] “Thorlabs DC2200 LED Driver.” [Online]. Available: https://www.thorlabs.com/thorproduct.cfm?partnumber=DC2200.

[16] "Thorlabs Mounted LEDs." [Online]. Available: https://www.thorlabs.com/newgrouppage9.cfm?objectgroup_ID=2692.

[17] “Thorlabs APD120A/M - Si Avalanche Photodetector, 400 - 1000 nm, M4 Taps.”[Online]. Available: https://www.thorlabs.com/thorproduct.cfm?partnumber=APD120A/M.

[18] M. S. Saud, I. Ahmed, T. Kumpuniemi, and M. Katz, "Reconfigurable optical-radio wireless networks: Meeting the most stringent requirements of future communication systems," Trans. Emerg. Telecommun. Technol., vol. 30, no. 2, pp. 1-15, 2019.

[19] “American National Standard for Safe Use of Lasers." [Online]. Available: https://as sets.lia.org/s3fs-public/pdf/ansi-standards/samples/ANSI Z136.1_sample.pdf.

[20] "PM100D - Compact Power and Energy Meter Console, Digital 4" LCD." [Online]. Available: https://www.thorlabs.com/thorproduct.cfm?partnumber=PM100D

[21] Kissi, C., Särestöniemi, M., Pomalaza-Raez, C., Sonkki, M., \& Srifi, M. N. (2018, October). Low-UWB directive antenna for wireless capsule endoscopy localization. In EAI International Conference on Body Area Networks (pp. 431-442). Springer, Cham.

[22] Tuovinen, T., Yazdandoost, K. Y., \& Iinatti, J. (2012, March). Comparison of the performance of the two different UWB antennas for the use in WBAN on-body communication. In 2012 6th European Conference on Antennas and Propagation (EUCAP) (pp. 22713374). IEEE.

[23] Dielectric Properties. [Online] Available: https://www.itis.ethz.ch/virtual-population/tissue- properties/database.

[24] Asan, N. B., Hassan, E., Velander, J., Mohd Shah, S. R., Noreland, D., Blokhuis, T. J., ... \& Augustine, R. (2018). Characterization of the fat channel for intra-body communication at R-band frequencies. Sensors, 18(9), 2752.

[25] Särestöniemi, M., Raez, C. P., Kissi, C., Kumpuniemi, T., Sonkki, M., Hämäläinen, M., \& Iinatti, J. (2019, October). Fat in the Abdomen area as a propagation medium in WBAN applications. In EAI International Conference on Body Area Networks (pp. 175-187). Springer, Cham. 